\title{
LOS ESTILOS DE PROSA Y EL ENFOQUE CIENCIA-TECNOLOGÍA-SOCIEDAD EN TEXTOS UNIVERSITARIOS DE QUÍMICA GENERAL
}

\author{
Malaver, Manuel ${ }^{1}$; Pujol, Rafael ${ }^{1}$ y d'Alessandro Martínez, Antonio ${ }^{2}$ \\ ${ }^{1}$ Universidad Pedagógica Experimental Libertador. Instituto Pedagógico de Caracas \\ ${ }^{2}$ Universidad Central de Venezuela y Universidad Simón Bolivar \\ agujero1@hotmail.com \\ dalessaa@camelot.rect.ucv.ve
}

\begin{abstract}
Resumen. En este trabajo se estudiaron los estilos de prosa científica y el enfoque ciencia-tecnología-sociedad en textos de química de los cursos básicos universitarios en Venezuela, usando técnicas de análisis de contenido. Se encuentra que el estilo de prosa más utilizado es el conocimiento en ciencia y que se da poca importancia al enfoque ciencia-tecnología-sociedad .
\end{abstract}

Palabras clave. Estilos de prosa, análisis de contenido, textos de química, estructura atómica, conocimiento en ciencia.

Summary. In this work we studied prose structures and STS interactions in basic chemistry textbooks commonly used in the initial university courses given in Venezuela with techniques of analysis of content. We have found the most used prose structure to be the knowledge of science, showing little interest in STS interactions.

Keywords. Prose structures, analysis of content, chemistry textbooks, atomic structure, knowledge of science.

\section{INTRODUCCIÓN}

Los libros de texto han sido y continúan siendo el material curricular más utilizado para la enseñanza de las ciencias en todos los niveles educativos. Esta importancia se ve reflejada en las investigaciones que se han realizado en los últimos años acerca de las características que debe tener un libro de texto de ciencias, así como las variables que influyen en el aprendizaje a partir de su contenido y su organización (Otero, 1997). Diversos autores han examinado su legibilidad y las actividades que le plantea al estudiante, además de las relaciones entre los modelos teóricos y su representación gráfica (Bullejos de la Higuera, 1983; Jiménez, Prieto y Perales, 1997).

En los libros de texto, generalmente, se ha mostrado una imagen de la ciencia totalmente desconectada de aspectos culturales y humanísticos, lo que impide conocer cómo es la ciencia y cómo trabajan los científicos (Solbes y Vilches, 1995), motivo por el cual, en el presente trabajo, se analizaron, entre otros aspectos: la creencia de que la ciencia son simples descubrimientos que condujeron a una construcción de conocimientos; el enfoque formalista, en el que se consideran conocimientos acabados y definitivos en contraste con el carácter tentativo de la ciencia; la naturaleza experimental de la ciencia en contraposición a una visión puramente teórica.

Una manera de relacionar el contenido de los textos a los problemas reales es a través del enfoque ciencia-tecnología-sociedad (CTS), ya que promueve el interés de los estudiantes por conectar la ciencia con las aplicaciones tecnológicas y los fenómenos de la vida cotidiana, aborda las implicaciones sociales y éticas que el uso de la 
tecnología conlleva y permite adquirir una compresión de la naturaleza de la ciencia y del trabajo científico (Caamaño, 1995) además de que prepara ciudadanos alfabetizados, científica y tecnológicamente, capaces de tomar decisiones informadas y acciones responsables (Membiela, 1997). Con este enfoque se muestra una imagen más real de lo que es la ciencia, de cómo trabajan los científicos y de cómo las ciencias físico-químicas han influido en el desarrollo de la historia de la humanidad (Solbes y Vilches, 1992). En definitiva, los objetivos del enfoque CTS pueden resumirse en tres campos: el de la ciencia aplicada, el de la ciencia y la sociedad y el de la naturaleza de la ciencia (Caamaño, 1995). Por ello, se consideró importante investigar si en los textos aparecen contenidos relacionados al enfoque CTS.

A modo de ejemplo se pueden señalar algunos temas CTS: el hambre en el mundo y los recursos alimentarios, el crecimiento de la población, la calidad del aire y de la atmósfera, los recursos del agua, la tecnología de guerra, la escasez de energía, el uso del suelo, las sustancias peligrosas, las reacciones nucleares y los recursos minerales (Bybee y Mau, 1986).

Debido a la importancia que tiene el estudio de la estructura de la materia en los cursos de química básica a nivel universitario y a las dificultades asociadas a su comprensión, es necesario conocer cómo se está abordando esta temática en los libros de texto de química general que se utilizan en este nivel de enseñanza. Se hace notar que existen muy pocos estudios hechos en textos de química de educación superior con relación a la estructura de la materia, razón adicional que justifica esta investigación. Asimismo, el impacto que ha tenido el desarrollo de la teoría atómica sobre la concepción moderna de la química ha modificado la visión del mundo actual, por lo que es imprescindible incorporarla como una de las temáticas más importantes de la educación universitaria en ciencias e ingeniería.

En los capítulos dedicados a la enseñanza de la estructura de la materia, los siguientes temas se encuentran relacionados con el enfoque CTS: emisión atómica, alumbrado público, utilidad del láser, superconductividad, isótopos radioactivos, rayos $\mathrm{X}$, resonancia magnética nuclear, biografías de científicos que hicieron descubrimientos relacionados con el comportamiento y la estructura de la materia (Malaver, 2001).

A pesar de la gran importancia que se atribuye al enfoque CTS en la educación científica, hay muy poco espacio dedicado a las interacciones CTS en los libros de texto de ciencias. Es decir, los textos de ciencias presentan escasa información acerca de cómo los científicos, ingenieros y técnicos adquieren un nuevo conocimiento y lo aplican para resolver problemas reales (Chiappetta, Fillman y Sethna, 1993; Fernández et al., 2002).

Otro aspecto que hay que tener en cuenta al elaborar textos es la relevancia de la historia de las ciencias y sus implicaciones en la formación científica. En la actualidad existe una opinión casi unánime entre los investigadores en educación sobre la importancia de la perspectiva histórica en la enseñanza de las ciencias. En los últimos años se ha verificado una progresiva incorporación de la historia de las ciencias tanto a la teoría como a la práctica de la enseñanza de las ciencias. De este modo, se produce un acercamiento entre áreas del conocimiento tradicionalmente ajenas entre sí, según una antigua clasificación que separa las ciencias de las humanidades (Lombardi, 1997).

La incorporación de la historia de la ciencia en la enseñanza de las mismas permite mostrar la ciencia como una construcción humana, colectiva, fruto del trabajo de muchas personas y no como una actividad hecha básicamente por genios, en su mayoría hombres. Presenta el carácter tentativo de la ciencia, las limitaciones de sus teorías, los problemas pendientes de solución, evitando visiones dogmáticas (Solbes y Traver, 1996). Para muchos docentes, enseñar ciencias es enseñar contenidos científicos y procedimientos propios de la formación de científicos e ingenieros y no enseñar una ciencia que contribuya a la formación general de todos los ciudadanos (Solbes y Traver, 2001).

En definitiva, es importante que en los libros de texto se incorporen aspectos históricos y filosóficos que puedan promover una enseñanza más crítica y humana, además de permitir que tanto el estudiante como el docente tengan una idea más elaborada de lo que es realmente la ciencia y de cómo trabajan los científicos (Matthews, 1994).

Adicionalmente, uno de los aspectos que permite establecer cuáles son los contenidos que el autor de un libro considera relevantes son las actividades y preguntas que él propone a lo largo del texto. Las preguntas y actividades pueden colocar a los estudiantes en la presencia de nuevas situaciones y los estudiantes deben definir un problema, identificar el conocimiento necesario para su solución y aplicar los principios y conceptos estudiados en el texto (Dreyfus, 1992). Debido a la importancia que presenta incluir actividades en donde se muestren las complejas relaciones entre ciencia, técnica y medio social y natural en que se insertan, se consideró relevante estudiar la frecuencia con la que aparecen, en los libros de texto analizados, las actividades y preguntas propuestas referidas al enfoque CTS.

El objetivo de esta investigación documental es analizar los estilos de prosa más utilizados por los textos universitarios de química general y el enfoque CTS que proyectan estos libros en el contenido sobre la estructura de la materia, tema fundamental en todo curso introductorio de química a nivel universitario, ya que contiene los conceptos básicos para la correcta compresión de los fenómenos físicos y químicos.

\section{MATERIALES Y MÉTODOS}

\section{Técnica de análisis de contenido}

Esta investigación se ubicó en la modalidad de estudio descriptivo, ya que se utilizaron técnicas de análisis de contenido en libros de texto, que permiten la recopila- 
ción de datos a fin de estudiar las características de dicho contenido, clasificando sus partes de acuerdo a categorías establecidas por el investigador (Ander-Egg, 1980; Ruiz Olabuenaga, 1996).

El análisis de contenido conforma tres etapas principales (Ander-Egg, 1980), a saber:

1) Establecer unidades de análisis, que no son más que los fragmentos que se toman como elementos para la investigación. En este trabajo se escogió como unidad de análisis la sección, la cual, según Strube (1989), es una unidad de prosa que incluye una sola idea. El mismo autor señala que las secciones usualmente están identificadas en el texto por marcas tipográficas como, por ejemplo, encabezamientos en letra cursivas o por subencabezados, indicando con ello una clara separación física entre las secciones, y quedan a juicio del investigador delimitar dónde comienza y termina una sección.

2) Determinar las categorías de análisis de las que depende la clasificación y la selección de la información buscada.

3) Seleccionar la muestra del material a ser analizado.

Para el análisis, se establecieron tres categorías de estilos de prosa, teniendo en cuenta los criterios sugeridos por Chiappetta y otros (1991a, 1991b), Pujol (1993) y Strube (1989). Éstas son:

1) Conocimiento en ciencia: En este estilo de prosa se presenta el contenido en términos de conceptos, hipótesis, principios, leyes y teorías, sin reflejar el proceso de búsqueda científica que condujo a tales conocimientos.

2) Naturaleza del conocimiento científico: Se refleja la actividad de indagación de la ciencia, que implica métodos y procesos tales como: observar, medir, clasificar, inferir, recordar datos, hacer cálculos, realizar experimentos, desarrollar modelos, hipótesis, leyes, etc. Asimismo, se presenta el desarrollo epistemológico de la ciencia.

3) La interrelación ciencia-tecnología-sociedad: En este estilo de prosa se representa el impacto de la ciencia y la tecnología en la sociedad y el ambiente. Se discuten y muestran algunos ejemplos de aplicaciones de la ciencia y de cómo el uso que se dé a la tecnología ayuda o perjudica a la humanidad. Se determinó también el porcentaje de actividades y preguntas propuestas referidas al enfoque CTS que aparecen en los capítulos dedicados a la estructura de la materia.

La información obtenida sobre los estilos de prosa permitió establecer algunas conclusiones referidas a la importancia que se da al enfoque CTS en los libros de texto, o si el contenido se presenta sustentado en argumentos o explicaciones científicas.

\section{Muestra bibliográfica}

Para delimitar la muestra de libros a ser analizados, se realizó una consulta a docentes del área universitaria con experiencia en química básica acerca de los textos más usados por la comunidad estudiantil. Con esta información, se elaboró una encuesta que fue aplicada en librerías del área metropolitana de Caracas que presentan un alto volumen de ventas.

En definitiva la muestra quedó conformada por los siguientes libros de texto:

Brown, T.L. y Le May, E. (1993). Química: la ciencia central. México DF: Prentice-Hall. Hispanoamérica, SA.

Chang, R. (1995). Química. México, DF: McGraw-Hill Interamericana, SA.

Masterton, W., Slowinski, E. y Staniski, C. (1989). Química general superior. México, DF: McGraw-Hill Interaméricana, SA.

Mahan, B. y Myers, R. (1990). Química: curso universitario. Wilmington, EEUU: Adison-Wesley Iberoamericana, $\mathrm{SA}$.

Mortimer, C. (1983). Química. México, DF: Grupo Editorial Iberoamérica.

En lo sucesivo, todas las referencias acerca de estos textos aparecerán de acuerdo con la denominación sugerida por Sanger y Greenbowe (1999), identificándolos con las iniciales de los autores. Siguiendo el orden anterior, los libros se identificaron como BL, Ch, MSS, MM y M.

\section{PRESENTACIÓN Y ANÁLISIS DE LOS RESUL- TADOS}

En la tabla I se muestran los diferentes criterios de análisis utilizados en el estudio del estilo de prosa en los textos universitarios de química general. Cada categoría se clasifica en una serie de subcategorías o criterios de análisis. Para la categoría «Conocimiento en ciencia», los criterios de análisis hacen énfasis en aspectos tales como: la presentación del contenido en los textos como un cuerpo de conocimientos acumulativos, la visión formalista de la ciencia y el uso de analogías en los textos. En la categoría «Naturaleza del conocimiento», se toman en cuenta los siguientes criterios: desarrollo histórico de conceptos y teorías científicas, carácter tentativo de la investigación, naturaleza empírica de la ciencia, elaboración de modelos científicos y evidencias esperimentales que apoyen o invaliden un modelo científico. Para la categoría de análisis «Interacción ciencia-tecnología-sociedad», se abordan las relaciones entre la ciencia, la tecnología y el medio social en el que se desenvuelven y el carácter colectivo de la actividad científica. Se presenta a continuación el significado de cada criterio de análisis utilizado en el estudio del estilo de prosa para cada categoría escogida: 
Tabla I

Criterios de análisis utilizados en el estudio del estilo de prosa de los textos de química básica a nivel universitario.

\begin{tabular}{|c|c|}
\hline Categoría & Criterio de análisis \\
\hline 1) Conocimiento en ciencia & $\begin{array}{l}\text { 1a: Resultados con explicaciones científicas } \\
\text { 1b: Conceptos científicos sustentados en hechos experimentales } \\
\text { 1c: Conocimientos científicos como producto de crisis y cambios profundos } \\
\text { de paradigmas } \\
\text { 1d: Uso de ejemplos numéricos } \\
\text { 1e: Uso de analogías }\end{array}$ \\
\hline 2) Naturaleza del conocimiento & $\begin{array}{l}\text { 2a: Desarrollo histórico de conceptos y teorías científicas } \\
\text { 2b: Carácter tentativo de la investigación } \\
\text { 2c: Naturaleza empírica de la ciencia } \\
\text { 2d: Elaboración de modelos científicos } \\
\text { 2e: Evidencias experimentales que apoyen o refuten un modelo científico }\end{array}$ \\
\hline 3) Interacción ciencia-tecnología-sociedad & $\begin{array}{l}\text { 3a: Tratamiento detenido de las relaciones ciencia-tecnología } \\
\text { 3b: Ciencia como una fuerza productiva o destructiva } \\
\text { 3c: Papel de la ciencia en la evolución de las ideas } \\
\text { 3d: Papel de la ciencia en la modificación del medio } \\
\text { 3e: Ciencia como fruto del trabajo colectivo } \\
\text { 3f: Valoración crítica del papel de la ciencia }\end{array}$ \\
\hline
\end{tabular}

\section{Conocimiento en ciencia}

Criterio la: Se muestra la manera cómo se llega a una teoría o postulado. Si se presentan los hallazgos y observaciones experimentales que permitieron formular una teoría en particular.

Criterio 1b: Las teorías o postulados establecidos están lo suficientemente sustentados en hechos y observaciones experimentales; es decir, si se explican los experimentos que conllevaron al desarrollo de una teoría o postulado.

Criterio 1c: Los conocimientos científicos se presentan como producto de crisis y de cambios profundos de paradigmas científicos.

Criterio 1d: Se utilizan ejemplos numéricos para apoyar o ilustrar la explicación de una teoría científica o postulado.

Criterio le: Se utilizan analogías que permitan clarificar algunos conceptos y teorías de cierta complejidad estableciendo similitudes con hechos o experiencias de la vida diaria.

\section{Naturaleza del conocimiento}

Criterio 2a: Se relaciona con el hecho de que, en la sección o fragmento analizado, la ciencia se considera como descubrimiento y no como una construcción de conocimientos, es decir, se muestra en la sección un planteamiento lineal y acumulativo del desarrollo científico en el que no aparecen la existencia de cambios conceptuales o de paradigma en las ideas aceptadas por los científicos.
Criterio 2b: Se toma en cuenta el papel de los problemas y dificultades en el desarrollo de la ciencia y los problemas que originaron la aparición de teorías importantes.

Criterio 2c: Se presenta en la sección o fragmento una visión empírica de la ciencia en el que los conocimientos científicos se forman a partir de observaciones y experimentos.

Criterio 2d: En los textos se muestra el desarrollo de los modelos científicos que permitieron explicar un fenómeno determinado, es decir, se hace referencia a la elaboración de los modelos científicos que conllevaron a establecer postulados como el modelo atómico de Bohr, el principio de incertidumbre de Heisenberg o el postulado de De Broglie.

Criterio $2 e$ : Se hace referencia a observaciones y hechos experimentales que apoyen o refuten un modelo científico propuesto para explicar un fenómeno determinado.

\section{Interacción ciencia-tecnología-sociedad}

Criterio $3 a$ : Se refiere a aspectos en los que el capítulo o fragmento analizado contiene tópicos en los cuales se relaciona la ciencia a los problemas reales y cotidianos, es decir, se presentan temas vinculados a las aplicaciones tecnológicas, como, por ejemplo, las aplicaciones del rayo láser, el desarrollo de la superconductividad y de la fibra óptica, el uso de isótopos radiactivos, etc.

Criterio 3b: Se hace énfasis en el poder de la ciencia para transformar los métodos de producción, para cambiar las relaciones sociales o para destruir regiones del planeta con armas nucleares, etc. 
Criterio 3c: Se hace énfasis en el papel que ha desempeñado la ciencia a lo largo de la historia en la evolución cultural de la humanidad; es decir, que aparezcan tópicos en los que se muestre que la evolución de la ciencia es un proceso en el que existen crisis, revoluciones, provocando cambios de paradigmas o bien que se incluyan en los capítulos analizados aspectos como la importancia de la ciencia en la evolución de las ideas filosóficas, artísticas, religiosas y las influencias de las ideologías en el desarrollo de la ciencia.

Criterio 3d: Se incluyen aspectos referidos al papel de la ciencia en la modificación del medio y problemas relacionados con cambios ambientales como el efecto invernadero, la destrucción de la capa de ozono, la lluvia ácida, etc.
Criterio 3e: Se muestra la ciencia como un producto colectivo, donde los logros y hallazgos se deben al trabajo de varios grupos de científicos.

Criterio 3f: Se muestran actividades en las que se señalan las consecuencias que tendrían para la humanidad las aplicaciones tecnológicas de la ciencia como el desarrollo y fabricación de reactores nucleares para fines pacíficos o la utilidad de los isótopos radiactivos en medicina.

En la tabla II se muestra cómo se distribuyen los diferentes criterios analizados de los estilos de prosa en las secciones que se refieren a la estructura de la materia de los textos universitarios de química general.

Tabla II

Frecuencia con la que aparecen los diferentes criterios de análisis que caracterizan a los estilos de prosa de los textos de química básica a nivel universitario.

\begin{tabular}{|c|c|c|c|c|c|c|c|c|c|c|}
\hline \multirow[t]{3}{*}{$\begin{array}{c}\text { Criterio } \\
\text { de análisis } \\
\text { (Ver tabla I) }\end{array}$} & \multicolumn{10}{|c|}{$\begin{array}{c}\text { Núm. de secciones que hacen referencia a ese criterio } \\
\text { Texto }\end{array}$} \\
\hline & \multicolumn{2}{|c|}{$\mathrm{Ch} \mathrm{N}_{\mathrm{T}}=72$} & \multicolumn{2}{|c|}{$\mathrm{BL} \mathrm{N}_{\mathrm{T}}=70$} & \multicolumn{2}{|c|}{$\operatorname{MSS} N_{T}=37$} & \multicolumn{2}{|c|}{$\mathrm{MMN}_{\mathrm{T}}=56$} & \multicolumn{2}{|c|}{$\mathrm{MN}_{\mathrm{T}}=42$} \\
\hline & $\mathrm{N}$ & $\%$ & $\mathrm{~N}$ & $\%$ & $\mathrm{~N}$ & $\%$ & $\mathrm{~N}$ & $\%$ & $\mathrm{~N}$ & $\%$ \\
\hline 1.a & 33 & 54,2 & 11 & 84,3 & 3 & 91,9 & 0 & 100 & 6 & 85,7 \\
\hline $\mathrm{b}$ & 36 & 50 & 36 & 51,4 & 20 & 54,1 & 40 & 71,4 & 29 & 69 \\
\hline $\mathrm{c}$ & 67 & 6,9 & 54 & 22,9 & 37 & 0,0 & 39 & 30,4 & 34 & 19 \\
\hline $\mathrm{d}$ & 45 & 62,5 & 42 & 60 & 25 & 67,6 & 31 & 55,4 & 15 & 35,7 \\
\hline $\mathrm{e}$ & 10 & 13,9 & 7 & 10 & 0 & 0 & 0 & 0 & 0 & 0 \\
\hline $2 a$ & 14 & 19,4 & 4 & 5,7 & 3 & 8,1 & 16 & 28,6 & 5 & 11,9 \\
\hline $\mathrm{b}$ & 11 & 15,3 & 5 & 7,1 & 3 & 8,1 & 24 & 42,9 & 4 & 9,5 \\
\hline $\mathrm{c}$ & 16 & 22,2 & 16 & 22,9 & 3 & 8,1 & 22 & 39,3 & 21 & 50 \\
\hline $\mathrm{d}$ & 20 & 27,8 & 33 & 47,1 & 19 & 51,4 & 38 & 67,9 & 23 & 54,8 \\
\hline $\mathrm{e}$ & 21 & 29,2 & 10 & 14,3 & 17 & 45,9 & 35 & 62,5 & 17 & 40,5 \\
\hline $3 a$ & 11 & 15,3 & 9 & 12,9 & 1 & 2,7 & 0 & 0 & 0 & 0 \\
\hline $\mathrm{b}$ & 1 & 1,4 & 0 & 0 & 0 & 0 & 0 & 0 & 0 & 0 \\
\hline $\mathrm{c}$ & 2 & 1,4 & 0 & 0 & 0 & 0 & 0 & 0 & 0 & 0 \\
\hline $\mathrm{d}$ & 5 & 2,8 & 1 & 1,4 & 0 & 0 & 0 & 0 & 0 & 0 \\
\hline $\mathrm{e}$ & 4 & 6,9 & 2 & 2,9 & 0 & 0 & 14 & 25 & 6 & 14,3 \\
\hline $\mathrm{f}$ & 4 & 5,6 & 3 & 4,3 & 0 & 0 & 18 & 32,1 & 7 & 16,7 \\
\hline
\end{tabular}

$\mathrm{N}=$ Número de secciones que presentan los diferentes criterios $\mathrm{N}_{\mathrm{T}}=$ Total de secciones en cada uno de los textos analizados 
Se observa que, en los cinco textos, el estilo de prosa que predomina es el «conocimiento en ciencia». Para el criterio 1a, los porcentajes obtenidos oscilan entre el $54,2 \%$ y el $91,9 \%$ para los cinco textos, donde el libro señalado como Ch alcanza un $54,2 \%$, resultado que indica que casi la mitad de las secciones en este texto presenta enunciados o resultados con explicaciones en los que se discute o analiza la forma en que se llegó a una ley, principio o concepto. En los textos restantes se observa que el número de secciones que tienen enunciados con explicaciones científicas es relativamente alto. Un ejemplo de párrafo que cumple con las características del criterio 1a es el siguiente tomado del libro $\mathrm{Ch}$ :

«[...] los físicos quedaron perplejos pero intrigados con la teoría de Bohr. La pregunta que planteaban acerca de ella era: ¿por qué el electrón en un átomo de Bohr está restringido a viajar en ciertas órbitas a distancias fijas del núcleo? Durante una década nadie, ni el mismo Bohr, tuvo una explicación lógica. En 1924 Louis de Broglie proporcionó una solución a este acertijo. De Broglie razonó como sigue: si las ondas luminosas se pueden comportar como un rayo de partículas (fotones), entonces quizás las partículas como los electrones pueden poseer propiedades ondulatorias. De acuerdo con De Broglie, un electrón enlazado a un núcleo se comporta como onda estacionaria. Las ondas estacionarias se pueden generar pulsando, por ejemplo, una cuerda de una guitarra [...]. Las ondas se describen como estacionarias porque no se desplazan a lo largo de una cuerda. Algunos puntos de la cuerda, llamados nodos, no se mueven en absoluto, esto es, la amplitud de la onda en estos puntos es cero. En cada extremo hay un nodo, y puede haber otros entre ellos. A mayor frecuencia de la vibración, menor es la longitud de la onda estacionaria y mayor el número de nodos. Como muestra la figura [...], sólo puede haber ciertas longitudes de onda en cualquiera de los movimientos permitidos de la cuerda.

»De Broglie argumentó que si el electrón se comporta en realidad como un onda estacionaria en el átomo de hidrógeno, entonces la longitud de onda debe caber exactamente en la circunferencia de la órbita Fig. [...]. De lo contrario, la onda se cancela en forma parcial a sí misma en cada circunvolución sucesiva; eventualmente la amplitud de la onda se reducirá a cero, y la onda no existiría.

»El razonamiento de De Broglie condujo a la conclusión de que las ondas se pueden comportar como partículas y éstas pueden exhibir propiedades ondulatorias» (p. 275).

En tres de los textos se observa un predominio del criterio 1c, referido a la presentación del conocimiento científico como producto de crisis y cambios de paradigmas (BL, MM y M). En el texto MM, este criterio aparece con una frecuencia de $30,4 \%$, lo que indica que el libro da cierta importancia a los criterios referidos a la naturaleza del conocimiento científico, en comparación con el resto de la muestra. Del mismo modo, el criterio $1 \mathrm{~b}$ en el texto MM presenta una frecuencia de $71,4 \%$, indicando con ello que, en este libro, se hace mucho énfasis en los hechos y evidencias experimentales para sustentar los conceptos e ideas científicas. Un ejemplo tomado del texto MM donde se presenta el criterio 1b: «En 1902 se sabía que la luz incidente sobre una superficie metálica limpia en el vacío hacía que la superficie emitiera electrones. La existencia de este efecto fotoeléctrico no era de sorprender; según la teoría clásica de la luz, era de esperar que la energía de una onda electromagnética se pudiera utilizar para extraer un electrón del metal. Pero la representación ondulatoria de la luz era totalmente incapaz de explicar los detalles del experimento. En primer lugar, los electrones no eran emitidos a menos que la frecuencia de la luz fuera mayor que cierto valor crítico $v$ como se muestra en la figura $[\ldots]$. Segundo, los electronés emitidos tenían energías cinéticas que aumentaban a medida que aumentaba la frecuencia de la luz, como se muestra en la figura [...]. Por último, aumentar la intensidad de la luz no cambiaba la energía de los electrones, pero aumentaba el número emitido por unidad de tiempo (pp. 453-454).

En los textos analizados se observa un bajo predominio del criterio 1c, el cual se refiere a que los conocimientos científicos se presentan como un producto de crisis y cambios profundos de paradigmas científicos. El caso más extremo es el texto MSS con $0,0 \%$.

En relación con el criterio 1d referido al uso de ejemplos numéricos, cuatro de los cinco textos estudiados (Ch, BL, MSS y MM) utilizan con más frecuencia ejemplos numéricos para ilustrar ideas científicas importantes, siendo el caso más destacado nuevamente el texto MSS, con un $67,6 \%$.

Para el criterio 1e (utilización de analogías), apenas se observa que dos textos (Ch y BL) utilizan estos recursos en sus explicaciones científicas, y con muy poca frecuencia $(10 \%$ y $14 \%$ con respecto al total de las secciones analizadas).

Con respecto al estilo denominado naturaleza del conocimiento científico, el criterio $2 \mathrm{a}$, que se refiere al desarrollo histórico de los conceptos y teorías científicas, aparece con más frecuencia en el texto MM con un $28,6 \%$, seguido del texto $\mathrm{Ch}$, con un $19,4 \%$. Se cita a continuación una sección del texto MM, en el que se da importancia al desarrollo histórico de los conceptos:

«Las primeras claves importantes sobre la naturaleza de la electricidad y de la estructura eléctrica de los átomos aparecieron en 1833 como resultado de las investigaciones de Faraday sobre la electrólisis. Sus hallazgos se pueden resumir en dos puntos:

»1) Una cantidad dada de electricidad siempre depositará sobre un electrodo el mismo peso de una sustancia dada.

»2) Los pesos de las distintas sustancias depositadas, desprendidas o disueltas en un electrodo por una cantidad fija de electricidad son proporcionales a los pesos equivalentes de estas sustancias.

»El contenido de los experimentos de Faraday fue reconocido en 1874 por G. J. Stoney, que fue el primero en 
sugerir el nombre de electrón para la partícula eléctrica fundamental. Sin embargo, hasta 1897 no se encontró ninguna evidencia experimental firme sobre la existencia y propiedades del electrón. La fuente de información decisiva fue la investigación de la conductividad eléctrica de los gases a presiones bajas» (pp. 443-444).

Con relación al criterio $2 \mathrm{~b}$, referido al carácter tentativo de la ciencia, el texto MM lo presenta con un $43 \%$, siendo este caso el de mayor frecuencia de los cinco textos. En los otros textos, este criterio no alcanza el 20\% del total de las secciones analizadas. Un ejemplo donde se muestra el criterio $2 \mathrm{~b}$ es el siguiente párrafo de MM:

«El modelo del átomo de Rutherford tenía un gran inconveniente: según todos los principios de la física conocidos en 1911, el átomo con un núcleo positivo tenía que ser inestable. Si los electrones eran estacionarios, no había nada que les impidiera ser atraídos hacia el núcleo; si estaban en movimiento circular, las bien fundamentadas leyes del electromagnetismo predecían que el átomo debería irradiar luz hasta que cesara todo movimiento electrónico. Sólo dos años después de la proposición de Rutherford, Niels Bohr intentó resolver esta aparente paradoja analizando la estructura atómica en función de la teoría cuántica de la energía, que había sido introducida por Max Planck en 1900. Antes de tratar las ideas de Bohr sobre el comportamiento de los electrones en los átomos, conviene examinar los experimentos que condujeron al desarrollo de los principios que utilizó Bohr» (p. 451).

El criterio 2c, referido a la visión empirista de la ciencia, aparece con más frecuencia en el texto $\mathrm{M}$, con un $50 \%$; seguido del texto MM, con un 39,3\%. Este mismo aspecto aparece con más frecuencia en el texto $\mathrm{M}$, con un $50 \%$; seguido del texto MM, con un 39,3\%. En los textos Ch y BL aparecen con una frecuencia del $22,2 \%$ y $22,9 \%$ respectivamente. Un ejemplo de este criterio es el siguiente párrafo tomado del texto $\mathrm{Ch}$ :

«Cada elemento tiene un espectro de emisión único. Las líneas características de los espectros atómicos se pueden utilizar en análisis químico para identificar átomos desconocidos, así como las huellas digitales sirven para identificar una persona. Cuando las líneas del espectro de emisión de un elemento conocido concuerdan exactamente con las líneas de un espectro de emisión de una muestra desconocida, la identidad de esta última se establece rápido. A pesar de que de inmediato se reconoció la utilidad de este procedimiento, el origen de estas líneas era desconocido a principios de este siglo. En 1913, no mucho después de los descubrimientos de Einstein y Planck, Niels Bohr ofreció una explicación teórica del espectro de emisión del átomo de hidrógeno» (p. 268).

Con relación al criterio $2 \mathrm{~d}$, referido a la elaboración de modelos científicos, en 3 libros aparece con una frecuencia superior al $50 \%$, siendo el texto $\mathrm{MM}$ el que presenta mayor frecuencia $(67,9 \%)$. En el texto BL, la frecuencia es cercana al $50 \%$ y llama la atención el libro $\mathrm{Ch}$ con apenas un $27,8 \%$ de frecuencia. Se cita a continuación una sección escogida del texto BL:
«Después que Rutherford descubrió la naturaleza nuclear del átomo, los científicos estuvieron tentados a considerar los átomos como sistemas solares microscópicos en los cuales los electrones orbitan al núcleo. No obstante, existía un problema con esta descripción, pues la física clásica predecía que debía emitirse radiación continua y que a medida que el electrón perdía energía se movería en espiral hacia el núcleo. Bohr resolvió este problema en forma muy semejante a la que Planck empleó para resolver el problema de la radiación del cuerpo negro. Consideró que la física clásica era inadecuada para describir los átomos. El modelo de Bohr consta de una serie de postulados que podemos resumir como sigue:

»1) El electrón de un átomo de hidrógeno se mueve alrededor del protón central en una órbita circular. Solamente están permitidas las órbitas de determinados radios, correspondientes a ciertas energías definidas. Se dice que un electrón en una de estas órbitas está en un estado de energía permitido. Un electrón en un estado de energía permitido no caerá en espiral hacia el núcleo.

»2) El electrón puede cambiar de un estado de energía permitido a otro, absorbiendo o emitiendo energía radiante. La frecuencia, $v$, de la energía radiante corresponde exactamente a la diferencia de energía, $\Delta \mathrm{E}$, entre los dos estados permitidos de energía: $\Delta \mathrm{E}=\mathrm{h} v \gg$ (pp. 200-201).

Con relación al criterio $2 \mathrm{e}$, que se refiere a presentar evidencias experimentales que apoyen o refuten un modelo científico, aparece con más frecuencia en el texto MM, con un $62,5 \%$. Un párrafo del texto $\mathrm{MM}$ en el que se muestre este criterio:

»Cuando se realizó por primera vez el experimento, Rutherford sabía que las partículas $\alpha$ eran átomos de helio doblemente ionizados de masa atómica 4; además, sus velocidades habían sido medidas por el citado método de la desviación magnética. En consecuencia, Rutherford sabía que la energía cinética de las partículas $\alpha$ era muy grande y comprendió que, para producir una desviación grande de una partícula tan energética, el átomo debía ser el asiento de una enorme fuerza eléctrica. También era evidente que esta fuerza debía ser ejercida por un cuerpo de masa considerable para que un cuerpo ligero como el electrón fuera arrastrado por la pesada partícula $\alpha$. Finalmente, el hecho de que solamente unas pocas partículas $\alpha$ experimentaban grandes desviaciones sugería que la gran fuerza eléctrica estaba confinada en regiones muy pequeñas del espacio que no eran alcanzadas por la mayoría de las partículas $\alpha$. En otras palabras, en lugar de ser una esfera de masa y densidad de carga uniforme, como había propuesto Thomson, el átomo era altamente no uniforme. Aunque los electrones podían ocupar el volumen asociado con la dimensión del átomo de $\sim 10^{-8} \mathrm{~cm}$, la electricidad positiva tenia que estar concentrada en un núcleo muy pequeño, pero pesado» (p. 449).

Por otra parte, el estilo de prosa denominado interacción ciencia-tecnología-sociedad no alcanza el $20 \%$ en los criterios 3a, 3b, 3c y 3d (Tabla I) para los cinco libros de texto. No ocurre así con el criterio $3 \mathrm{f}$, que se refiere a la valoración crítica del papel de la ciencia, el cual supera el $30 \%$ en el texto MM. 
Tabla III

Frecuencia total con la que aparecen los diferentes criterios de análisis en los cinco libros analizados.

\begin{tabular}{|ccc|}
\hline $\begin{array}{c}\text { Criterio analizado } \\
\text { (Ver Cuadro 1b) }\end{array}$ & \multicolumn{2}{c|}{ Frecuencia con la que aparece el criterio } \\
$\mathbf{N}_{\mathbf{T}}=\mathbf{2 7 7}$
\end{tabular}

$\mathrm{N}_{\mathrm{T}}=$ Número total de secciones analizadas

$\mathrm{N}=$ Número total de secciones que presentan los diferentes criterios

En la tabla III se muestra el porcentaje total de secciones que contienen aspectos de los estilos de prosa. Se observa un fuerte predominio del estilo denominado conocimiento en ciencia con respecto a los otros dos estilos. Apenas se toma en cuenta en los libros analizados la interacción CTS, por lo que no aparece como una parte integral de los textos.

En la tabla IV se indican los contenidos referidos al enfoque CTS que aparecen en los capítulos de la estructura de la materia.

Siguiendo la clasificación que han hecho Bybee y Mau (1986) y Caamaño (1995) sobre temas CTS se observa que casi todos los contenidos corresponden a aplicaciones y desarrollos tecnológicos de los diversos descubrimientos y teorías en el campo de la estructura de la materia. El aspecto 7 referido a la biografía un poco detallada y obra de los científicos se eligió teniendo como base las investigaciones de Solbes y Traver (1996) y Solbes y Vilches (1989), en las que se establece que la ciencia no es fruto del trabajo de unos genios, hombres por lo general, sino que es producto de un esfuerzo colectivo y humano de hombres y mujeres. La presencia de biografías de científicos y sus descubrimientos permite mostrar al lector la contribución de los hombres de ciencia en la evolución de las ideas, las crisis de paradigmas y los cambios conceptuales en las ideas aceptadas por los científicos (Solbes y Traver, 1996).

El libro Ch es el que tiene el mayor número de estos contenidos, mostrando las diversas aplicaciones tecnológicas de la temática presentada. El aspecto que se incluye en todos los textos es el del uso del espectrómetro de masas y de los rayos $\mathrm{X}$.

En la tabla V se muestra la frecuencia con la que aparecen en los textos las actividades y preguntas propuestas relacionadas con el enfoque CTS. Se observa que sólo los textos $\mathrm{Ch}$ y BL presentan este tipo de preguntas pero con frecuencias que apenas llegan al $1 \%$ y $0,8 \%$ respectivamente. Estos libros son los que contienen el mayor número de actividades y preguntas en los capítulos analizados.

En los textos restantes (MSS, MM y M) se observa una total ausencia de preguntas o actividades referidas a este enfoque.

Los estilos de prosa científica, el enfoque CTS y las actividades y preguntas propuestas son buenos indicadores de la imagen de la ciencia que podría estarse proyectando en los capítulos referidos a la estructura de la materia de los textos analizados.

En la tabla III se observa que los estilos denominados «la naturaleza del conocimiento» y la «interacción cienciatecnología-sociedad» aparecen con baja frecuencia, predominando el estilo «conocimiento en ciencia».

Con respecto al contenido referido a la vida y obra de los científicos, ninguno de los textos analizados incluye este aspecto, presentando una imagen del científico como un genio que trabaja y descubre en solitario, contribuyendo con esto a mostrar una idea tergiversada de la ciencia y del trabajo de los científicos. 
Tabla IV

Contenidos referidos al enfoque ciencia, tecnología y sociedad que aparecen en los capítulos sobre la estructura de la materia en los textos de química básica a nivel universitario.

\begin{tabular}{|ccccccccc|}
\hline \multicolumn{7}{|c|}{ Contenidos referidos al enfoque CTS } \\
\hline Texto & 1 & 2 & 3 & 4 & 5 & 6 & 7 & $\%$ S \\
\hline Ch & S & S & S & S & N & N & N & 57,14 \\
BL & N & N & S & N & S & S & N & 42,86 \\
MSS & N & N & S & N & N & N & N & 14,30 \\
MM & N & N & S & N & S & N & N & 28,57 \\
M & N & N & S & N & N & N & N & 14,30 \\
\hline
\end{tabular}

$\mathrm{S}=$ Aparece el contenido

$\mathrm{N}=$ No aparece el contenido

1) Aplicación de la emisión atómica y del láser

2) Aplicaciones del microscopio electrónico

3) Usos del espectrómetro de masas y de los rayos $\mathrm{x}$

4) Desarrollo de la superconductividad y de la fibra óptica

5) Uso de los isótopos radiactivos

6) Usos de la resonancia magnética nuclear

7) Vida y obra de los científicos en el campo de la estructura atómica

$\% \mathrm{~S}=$ Porcentaje de contenido referido al enfoque CTS

Tabla V

Porcentaje de actividades y preguntas relacionadas con el enfoque CTS que aparecen en los capítulos referidos a la estructura de la materia en los libros de texto de química básica a nivel universitario.

\begin{tabular}{|cccc|}
\hline & Actividades y preguntas relacionadas con el enfoque C T S \\
\hline Texto & $\mathbf{N}_{\mathbf{T}}$ & $\mathbf{N}$ & \% \\
\hline Ch & 674 & 7 & 0,0 \\
BL & 388 & 3 & 0 \\
MSS & 312 & 0 & 0 \\
MM & 163 & 0 & 0 \\
M & 216 & 0 & 0 \\
\hline
\end{tabular}

$\mathrm{N}_{\mathrm{T}}=$ Total de preguntas y actividades del texto referidas a la estructura de la materia

$\mathrm{N}=$ Total de preguntas referidas al enfoque CTS

En cuanto a las actividades y preguntas propuestas, sólo dos textos contienen algunas preguntas referidas al enfoque CTS. En el texto BL no se da importancia a este tipo de enfoque.

Del análisis de la información obtenida en la presente investigación se establece que el estilo de prosa «Conocimiento en ciencia» es el más frecuentemente utilizado (Tabla II), indicando con ello que los conceptos propios de la química conforman la temática principal de los textos.

El texto MM da gran importancia a los criterios referidos a la naturaleza del conocimiento científico, mostrando el contexto histórico en el que se desarrollaron las teorías de la estructura atómica, lo que los otros textos prácticamente no mencionan.

Si bien es cierto que en todos los textos los resultados con explicaciones científicas están por encima del $50 \%$ (criterio 1.a), el texto $\mathrm{Ch}$ presenta una baja frecuencia con respecto a los otros libros. El texto Ch tiene un alto porcentaje de enunciados sin justificación. En efecto, este libro no justifica plenamente la teoría atómica de
Dalton, al presentar las hipótesis de este autor como hechos establecidos, sin apoyarse en ninguna evidencia experimental. Tópicos como la teoría cuántica de Planck, el principio de incertidumbre, la relación cargamasa del electrón, el modelo de Bohr aparecen sin una sólida justificación teórica y experimental en este texto. Por ejemplo, en el libro $\mathrm{Ch}$, al referirse al principio de incertidumbre de Heisenberg, tan sólo se menciona que fue una de las consecuencias más importantes de la dualidad onda-partícula y que fue formulado por este autor sin mostrar el experimento mental que fue la base del enunciado de este principio. Se cita a continuación el principio de incertidumbre tomado del texto $\mathrm{Ch}$ :

«Una de las consecuencias más importantes de la naturaleza dual del electrón es el principio de incertidumbre, el cual fue formulado por el físico alemán Werner Heisenberg. El principio de incertidumbre de Heisenberg establece que es imposible conocer simultáneamente el momento ( $p$, definido como el producto de la masa por la velocidad) y la posición $(x)$ de una partícula con certidumbre. Expresado en forma matemática, se escribe:

$$
\Delta \mathrm{x} \Delta \mathrm{p} \geq \mathrm{h} / 4 \pi
$$


donde $\Delta \mathrm{x}$ y $\Delta \mathrm{p}$ son las incertidumbres en las mediciones de posición y momentum, respectivamente» (p. 278).

Aunque los cinco textos de la muestra hacen referencia a la determinación experimental de la relación $\mathrm{e} / \mathrm{m}$ del electrón y a la carga del electrón, presentando estos descubrimientos como de una gran importancia en el desarrollo de la teoría de la estructura atómica, no se plantean los problemas y las controversias que formaron parte de estos hallazgos.

De acuerdo con Morrow (1969), para la época en que Millikan realiza el experimento de la gota de aceite, anteriores investigadores habían logrado medir la carga promedio del electrón obteniendo un valor muy cercano al aceptado. Millikan estableció que el electrón lleva siempre una carga definida e invariante. El mismo autor señala que el primer intento de hacer una determinación directa de la carga del electrón fue el de un estudiante de Thomson, J. Townsend, entre 1897 y 1898, quien fue precursor de las técnicas experimentales que se usaron para determinar las cargas de partículas subatómicas y atómicas. Townsend reporta el valor de $3,1 \times 10^{-10}$ u.e.e (unidades electrostáticas) para las partículas de carga negativa.

Morrow (1969) también afirma que Wilson, en 1903, obtiene el mismo valor de $3,1 \times 10^{-10}$ u.e.e. y que Millikan y Begeman reportan el valor de $4,06 \times 10^{-10}$ u.e.e. para la carga del electrón, estableciendo este valor como la unidad de carga fundamental e invariante.

Con respecto a la determinación de la relación carga-masa (e/m) del electrón, no se mencionan los estudios de Faraday sobre el paso de la electricidad a través de atmósferas gaseosas enrarecidas (Morrow, 1969; Garritz, 1997) ni la invención del tubo de rayos catódicos por Plücker y Geissler (Garritz, 1997). Ningún texto hace referencia al hallazgo de Schuster con respecto al valor de la relación carga-masa de los rayos catódicos ni al verdadero valor numérico de la relación e/m que obtuvo Thomson, que fue de $1,76 \times 10^{11} \mathrm{C} / \mathrm{Kg}$.

Con respecto al estilo denominado interacción cienciatecnología-sociedad», los textos donde se observa una mayor frecuencia para los criterios de este estilo son los libros Ch y MM.

El criterio 3a, referido al tratamiento de las relaciones ciencia-tecnología, se presenta con una frecuencia de $15,3 \%$ en el texto $\mathrm{Ch}$, y $12,9 \%$ en el texto BL, sin alcanzar el $5 \%$ en los otros libros. En estos textos se observa una preocupación por parte del autor en presentar algunas aplicaciones básicas de la ciencia, que es uno de los objetivos del enfoque CTS, mostrando temas interesantes como los usos de la resonancia magnética nuclear y el microscópico electrónico, el láser en medicina e ingeniería, la emisión atómica, el alumbrado público... entre otros.

En el texto MM, el criterio 3e aparece con una frecuencia superior al $20 \%$. Esto indica la importancia que otorga el autor a las referencias históricas y a la ciencia como pro- ducto del trabajo colectivo al intentar ofrecer una imagen de la ciencia llena de controversias y discusiones entre grupos de científicos, tomando en cuenta los aspectos históricos de la misma. Un párrafo del texto MM donde se muestre la ciencia como fruto del trabajo colectivo es el siguiente:

«El trabajo de Bohr fue la primera aplicación de la hipótesis cuántica a la estructura atómica que tuvo cierto éxito. Téngase en cuenta, sin embargo, que la teoría de Bohr era incorrecta; fue abandonada doce años más tarde a favor de la actual teoría cuántica de la estructura atómica. A pesar de esto, las ideas de Bohr tenían bastante fundamento para permitirle explicar por qué únicamente ciertas frecuencias de luz eran irradiadas por los átomos $\mathrm{y}$, en algunos casos, pronosticar exactamente los valores de estas frecuencias. Además, las propuestas de Bohr ayudaron considerablemente a Moseley a comprender sus mediciones de las frecuencias de los rayos X emitidos y a utilizarlas para determinar los números atómicos. Así pues, aunque luego fue abandonada, esta teoría inicial fue un paso importante para la comprensión de la estructura atómica» (p. 454).

Con respecto al uso de analogías (aspecto 1e) del estilo «conocimiento en ciencia»se observa que sólo los textos Ch y BL utilizan analogías cuando hacen referencia a algunas explicaciones científicas.

En el texto BL, cuando el autor explica el modelo de repulsión de pares electrónicos del nivel de valencia, hace una analogía entre el acomodo de los pares electrónicos y el arreglo espacial de globos a todos en sus extremos. Esta analogía permite clarificar al lector el modelo RPENV al establecer similitud de estos fenómenos con hechos o acontecimientos de la vida cotidiana. Al respecto, Duit (1991) establece que las analogías son herramientas muy útiles que facilitan el proceso de aprendizaje de conceptos básicos y que deben considerarse como un aspecto esencial de la enseñanza de la ciencia.

La información representada en la tabla III permite afirmar que, en general, los textos analizados muestran una fuerte tendencia a centrarse en la definición de contenidos, principios, leyes y teorías propios de la química, otorgando poca importancia a presentar las aplicaciones tecnológicas de la ciencia, su papel en la evolución de la sociedad y la naturaleza de la ciencia.

Por otra parte, en la tabla IV se observa que el texto Ch presenta el mayor número de aspectos relacionados con las aplicaciones tecnológicas de los contenidos de la estructura de la materia. En este libro se incluyen temas como las aplicaciones de la emisión atómica y el láser, el microscopio electrónico y superconductividad y las fibras ópticas, tópicos que no aparecen en los otros textos de la muestra.

El texto BL, aunque no contiene un gran número de temas relacionados con el enfoque ciencia, tecnología y sociedad, incluye un espacio dedicado a las aplicaciones de la resonancia magnética nuclear, aspecto que no se menciona en los restantes libros. 
Con respecto a la frecuencia con que aparecen las actividades y preguntas relacionadas con este enfoque, el mayor porcentaje se observa en el texto $\mathrm{Ch}$, que corresponde a un $1 \%$ del total de preguntas analizadas para este libro. El texto BL apenas muestra una frecuencia de 0,8\% para 388 preguntas. Los libros restantes no contienen ningún tipo de actividades referidas a este enfoque. Ejemplo de este tipo de preguntas que aparecen en los textos BL y Ch son las siguientes:

«a) La teoría cuántica considera que los cambios de energía no son continuos. ¿Por qué no apreciamos este efecto en nuestras actividades de la vida diaria?»(BL, p. 233);

$\gg b)$ las tuberías de agua que están a la intemperie se tienen que drenar o aislar en invierno en los climas fríos. ¿Por qué?» (Ch, p. 491).

Es interesante hacer referencia al hecho de que el texto $\mathrm{Ch}$ es el que presenta el mayor número de tópicos relacionados al enfoque CTS; sin embargo, no contiene un número apreciable de preguntas relacionadas con los aspectos tecnológicos de la química.

En la tabla VI se muestran las características más importantes de los cinco libros de texto analizados. Se observa que, aunque en el texto MM el estilo de prosa predominante es el conocimiento en ciencia, hay un gran interés por parte del autor en mostrar el desarrollo histórico de los conceptos y el carácter tentativo de la ciencia.

Los resultados anteriores permiten afirmar que, aunque los libros de texto presenten tópicos pertenecientes al enfoque CTS, muestran muy poca inclinación por el desarrollo de estos temas y dan mayor importancia a los contenidos de tipo científico, aun cuando el movimiento
CTS surgió en los años sesenta y setenta en el medio universitario (Membiela, 1997) .

El análisis de los estilos de prosa científica, del enfoque ciencia-ambiente-tecnología y sociedad y de las preguntas y actividades indican que en la enseñanza de la estructura de la materia se ignoran los aspectos de tipo histórico, por lo que los alumnos reciben una imagen deformada sobre la evolución y el avance de los conceptos científicos.

Los textos utilizan escasamente algunos recursos históricos como breves biografías o narración de hallazgos o descubrimientos y muestran en los capítulos dedicados a la estructura del átomo la historia de algunos conceptos y modelos.

Por otra parte, en los textos se ignoran las situaciones conflictivas que originaron las investigaciones científicas y que modificaron los modelos científicos. En la mayoría de los textos (Ch, BL, MSS, y M) no se observa un intento por parte del autor en mostrar la ruptura con la filosofía positivista, en la que se establece que el mundo consiste en hechos positivos y reales y en fenómenos observables, y el conocimiento científico existe sólo en la descripción de estos fenómenos (Níaz, 1994), lo que no ocurre cuando se plantean aspectos como el principio de incertidumbre de Heisenberg y la dualidad onda-partícula, que son consecuencia de la aplicación del método hipotético-deductivo al estudio de la estructura atómica. Sólo el texto MM hace un intento de explicar este cambio de paradigma científico.

En general, los libros de texto no realizan un tratamiento detenido de las relaciones ciencia-técnica, por lo que se ofrece a los alumnos una imagen de la ciencia desconectada de los problemas reales y cotidianos.

Tabla VI

Características más importantes de los cinco libros de texto analizados.

\begin{tabular}{|c|c|c|c|c|c|}
\hline $\begin{array}{l}\text { Características } \\
\text { Estilo de prosa }\end{array}$ & Chang & Brown - Le May & $\begin{array}{c}\text { Masterton } \\
\text { Slowinski-Staniski }\end{array}$ & Mahan-Myers & Mortimer \\
\hline $\begin{array}{l}\text { 1) Conocimiento } \\
\text { en ciencia }\end{array}$ & $\begin{array}{l}\text { Contenido como } \\
\text { conceptos, principios, } \\
\text { leyes }\end{array}$ & $\begin{array}{l}\text { Contenido como } \\
\text { conceptos, principios, } \\
\text { leyes }\end{array}$ & $\begin{array}{l}\text { Contenido como } \\
\text { conceptos, principios, } \\
\text { leyes }\end{array}$ & $\begin{array}{l}\text { Más importancia } \\
\text { a la naturaleza del } \\
\text { conocimiento }\end{array}$ & $\begin{array}{l}\text { Más importancia } \\
\text { a la naturaleza } \\
\text { del conocimiento }\end{array}$ \\
\hline $\begin{array}{l}\text { 2) Naturaleza } \\
\text { del conocimiento }\end{array}$ & $\begin{array}{l}\text { Poca importancia } \\
\text { a la naturaleza } \\
\text { del conocimiento }\end{array}$ & $\begin{array}{l}\text { Poca importancia } \\
\text { a la naturaleza del } \\
\text { conocimiento }\end{array}$ & $\begin{array}{l}\text { Muy poca importancia } \\
\text { a la naturaleza } \\
\text { del conocimiento }\end{array}$ & $\begin{array}{l}\text { Gran interés } \\
\text { en mostrar } \\
\text { la naturaleza } \\
\text { del conocimiento }\end{array}$ & $\begin{array}{l}\text { Hay poco interés } \\
\text { en mostrar } \\
\text { el desarrollo } \\
\text { de los conceptos } \\
\text { y el carácter } \\
\text { tentativo } \\
\text { de la ciencia }\end{array}$ \\
\hline $\begin{array}{l}\text { 3) Interacción ciencia- } \\
\text { tecnología-sociedad }\end{array}$ & $\begin{array}{l}\text { Poco interés en } \\
\text { presentar este estilo }\end{array}$ & $\begin{array}{l}\text { Poca importancia a la } \\
\text { interacción CTS }\end{array}$ & No está contenido & $\begin{array}{l}\text { Poco interés } \\
\text { en presentar } \\
\text { este aspecto }\end{array}$ & $\begin{array}{l}\text { Se da muy } \\
\text { poca importancia } \\
\text { a este estilo }\end{array}$ \\
\hline
\end{tabular}




\section{CONCLUSIONES Y PERSPECTIVAS}

Los resultados obtenidos en el análisis del contenido referido a la estructura de la materia en los textos universitarios de química general permitieron llegar a las siguientes conclusiones:

- El estilo de prosa más comúnmente usado por los textos analizados es el conocimiento en ciencia, en el que se presenta el contenido científico en términos de conceptos, principios, leyes y teorías.

- En la mayoría de los textos de la muestra se hace muy poco énfasis en los contenidos referidos al enfoque CTS, mostrando con ello una ciencia desconectada del contexto social y tecnológico del que forma parte. Asimismo, los textos muestran un bajo porcentaje de actividades y preguntas relacionadas con este enfoque, apenas un 0,6 $\%$ del total de las preguntas, lo que indica la poca importancia que le otorgan los autores de los textos a preguntas que impliquen el uso de la información disponible para plantear alternativas de solución a un problema de la comunidad.

- El hecho de que aparezca con más frecuencia el estilo de prosa denominado «conocimiento en ciencia» indica que, en los textos, se ofrece una imagen de la ciencia que consiste en una acumulación de conocimientos, en el que no se muestran los conflictos de los grandes paradigmas científicos que producen cambios de conceptos y modelos. Asimismo, no se toma en cuenta el contexto histórico y social del que forman parte las teorías científicas.
- Los textos deben hacer mayor énfasis en el papel que puede desempeñar la historia de la ciencia en la enseñanza de la misma. De esta manera se toman en cuenta los problemas históricos que originaron el desarrollo de las grandes teorías científicas y las hipótesis que se plantearon en su elaboración.

Debe darse más importancia, en los textos, al enfoque CTS, por lo que se recomienda que los libros de texto:

- Muestren la ciencia como una actividad humana y colectiva, producto del esfuerzo de muchas personas, evitando de este modo la imagen de una ciencia hecha por científicos geniales que trabajan en solitario, hombres en su mayoría.

- Realicen un tratamiento más detallado de las relaciones ciencia-tecnología-sociedad, mostrando de este modo las aplicaciones tecnológicas de la ciencia.

- Expongan el papel que ha desempeñado la ciencia en el contexto histórico y las influencias políticas y sociales en el desarrollo científico.

- Presenten actividades y preguntas relacionadas con las relaciones ciencia-tecnología.

- Indiquen referencias de tipo biográfico de algunos científicos, donde aparezca el contexto histórico y social en el que vivieron, así como también sus descubrimientos. 


\section{REFERENCIAS BIBLIOGRÁFICAS}

ANDER-EGG, E. (1980). Técnicas de investigación social. Buenos Aires: El Cid Editor.

BYBEE, R.W. y MAU, T. (1986). Science and technology related global problems. International Survey of Science Educators. Journal of Research Science Teaching, 23(7), pp. 599-618.

CAAMAÑO, A. (1995). La educación ciencia-tecnologíasociedad: una necesidad en el diseño del nuevo curriculum de ciencias. Alambique. Didáctica de las Ciencias Experimentales, 3, pp. 4-6.

CARMEN, L. y JIMÉNEZ P. (1997). Los libros de texto: un recurso flexible. Alambique. Didáctica de las Ciencias Experimentales, 11, pp. 7-14.

CHIAPPETTA, E.L., FILLMAN, D.A. y SETHNA, G.H. (1991a). A method to quantify major themes of scientific literary in science textbooks. Journal of Research in Science Teaching, 28(8), pp. 713-725.

CHIAPPETTA, E.L., FILLMAN., D.A. y SETHNA, G.H. (1991b). A quantitative analysis of high school chemistry textbooks for scientific literary themes and expository learning aids. Journal of Research in Science Teaching, 28(10), pp. 939-954.

CHIAPPETTA, E.L., FILLMAN, D.A. y SETHNA, G.H. (1993). Do middle school life science textbooks provide a balance of scientific literary themes? Journal of Research in Science Teaching, 30(7), pp. 787-797.

DREYFUS, A. (1992). Contest analysis of school textbooks. The case of technology-oriented curriculum. International Journal of Science Education, 14(1), pp. 3-12.

DUIT, R. (1991). On the role of analogies and metaphors in learning science. Science Education, 75(6), pp. 649-672.

FERNÁNDEZ, I., GIL, D., CARRASCOSA, J., CACHAPUZ, A. y PRAIA, J. (2002). Visiones deformadas de la ciencia transmitidas por la enseñanza. Enseñanza de las Ciencias, 20(3), pp. 477-488

GARRITZ, A. (1997). El electrón centenario. Educación química, 8(3), pp. 114-117.

IZQUIERDO, M. y RIVERA, L. (1997). La estructura y la comprensión de los textos de ciencia. Alambique. Didáctica de las Ciencias Experimentales, 11, pp. 24-33

KAMSAR, J.W. (1987). Utilizing a historical perspective in the teaching of Chemistry. Journal of Chemical Education, 64(11), pp. 931-932.

LOMBARDI, O.L. (1997). La pertenencia de la historia en la enseñanza de la ciencias: argumentos y contra argumentos. Enseñanza de las Ciencias, 15(3), pp. 343 -349.
MALAVER, M. (2001). «Análisis del contenido referido a la estructura de la materia en libros de texto de química básica a nivel universitario». Tesis de maestría. Universidad Pedagógica Experimental Libertador. Instituto Pedagógico de Caracas.

MATTHEWS, M.R. (1994). Historia, filosofía y enseñanza de las ciencias: la aproximación actual. Enseñanza de las Ciencias, 12(2), pp. 255 -277.

MEMBIELA, P. (1997). Una revisión del movimiento educativo Ciencia-Tecnología-Sociedad. Enseñanza de las Ciencias, 15(1), pp. 51-57.

MORROW, B. A. (1969). On the discovery of the electron. Jounal of Chemical Education, 46(9), pp. 584-588.

NÍAZ, M. (1994). Más allá del positivismo: una interpretación lakatosiana de la enseñanza de las ciencias. Enseñanza de las Ciencias, 12(1), pp. 97-100.

PUJOL, R. (1993). «Análisis del contenido, las ilustraciones y las actividades propuestas en la unidad referente a la estructura de la materia de los libros de texto en química para el noveno grado de educación básica». Trabajo de ascenso no publicado. Universidad Pedagógica Experimental Libertador. Instituto Pedagógico de Caracas.

RUIZ OLABUENAGA, J. L. (1996). Metodología de la investigación cualitativa. Bilbao: Universidad de Deusto.

SANGER, M. J. y GREENBOWE, T. J. (1999). An analysis of college chemistry textbooks and sources of misconceptions and errors in electro-chemistry. Journal of Chemical Education, 78(6), pp. 853-860.

SOLBES, J. y TRAVER, M. (1996). La utilización de la historia de las ciencias en la enseñanza de la física y la química. Enseñanza de las Ciencias, 14(1), pp. 103-112.

SOLBES, J. y TRAVER, M. (2001). Resultados obtenidos introduciendo historia de la ciencia en las clases de física y química: mejora de la imagen de la ciencia y desarrollo de actitudes positivas. Enseñanza de las Ciencias, 19(1), pp. 151-162.

SOLBES, J. y VILCHES, A. (1992). El modelo constructivista y las relaciones ciencia-técnica-sociedad (CTS). Enseñanza de las Ciencias, 10(2), pp.181-186.

SOLBES, J. y VILCHES, A. (1995). El profesorado y las actividades CTS. Alambique. Didáctica de las Ciencias Experimentales, 3, pp. 30-38.

STRUBE, P. (1989). A content analysis of argument and explanation present to students in physical science textbooks: a model and example. International Journal of Science Education, 11(2), pp. 195-202. 